\title{
The European Commission Organisation Environmental Footprint method: comparison with other methods, and rationales for key requirements
}

\author{
Nathan Pelletier • Karen Allacker • Rana Pant • \\ Simone Manfredi
}

Received: 6 September 2012 / Accepted: 29 May 2013 / Published online: 16 August 2013

(C) The Author(s) 2013. This article is published with open access at Springerlink.com

\begin{abstract}
Purpose The European Commission (EC) has developed a reference method for organisation environmental footprinting (OEF) in support of improving the sustainability of production and consumption. This methodological development was guided by four core criteria. Specifically, it was deemed necessary that the method provides for a (1) multi-criteria, (2) life cycle-based approach that considers all organisational and related activities across the supply chain, (3) provides for reproducibility and comparability over flexibility, and (4) ensures physically realistic modelling.

Methods Here, we review a subset of existing organisation environmental footprinting methods. We evaluate key areas of convergence (very limited!) and divergence between these methods, and the extent to which the methodological specifications they provide satisfy the four aforementioned criteria for the EC OEF method. On this basis, we specify where and why the EC OEF method necessarily diverges from and/or goes beyond the reviewed methods.

Results and discussion We found little consistency between the reviewed methods, and few instances where our four criteria for the EC OEF method were satisfied. We specify the methodological norms for the EC OEF method for, among other things, definition of the unit of analysis (the organisation) and reference flow; organisation and analytical boundaries; cut-off criteria; impact categories and models; allocation solutions; and data quality. We further provide a rationale for each norm, in particular why they diverge from the various options presented in the reviewed methods.
\end{abstract}

Responsible editor: Matthias Finkbeiner

N. Pelletier $(\bowtie) \cdot$ K. Allacker $\cdot$ R. Pant $\cdot S$. Manfredi

European Commission Directorate General-Joint Research Centre,

Institute for Environment and Sustainability, Ispra, Italy

e-mail: nathan.pelletier@jrc.ec.europa.eu
Conclusions In order to satisfy the four core criteria, the EC OEF method diverges from/goes beyond the reviewed methods in a variety of important respects. We suggest that the end result represents a significant advance in the standardization of life cycle-based organisation environmental footprinting.

Keywords Environmental footprint · European Commission $\cdot$ LCA $\cdot$ Organisation $\cdot$ Review

\section{Introduction}

The "Roadmap for a Resource Efficient Europe" comprises a key component of the European Commissions' "Europe 2020 Strategy". The central aim of this roadmap is to "increase resource productivity and to decouple economic growth from resource use and its environmental impact" (European Commission 2011a). A central challenge identified in the Roadmap is "transforming the economy onto a resource-efficient path that will bring increased competitiveness and new sources of growth and jobs through cost savings from improved efficiency, commercialisation of innovations and better management of resources over their whole life cycle" (European Commission 2011b). Four specific aspects are addressed: sustainable consumption and production; turning waste into a resource; supporting research and innovation; and removing environmentally harmful subsidies/getting the prices right.

In support of the sustainable production and consumption objective, the Roadmap set as a milestone to provide "the right incentives for citizens and public authorities to choose the most resource efficient products through appropriate price signals and clear environmental information." Towards this end, the European Commission (EC) was requested by Parliament to 
"establish a common methodological approach to enable Member States and the private sector to assess, display and benchmark the environmental performance of products, services and companies based on a comprehensive assessment of environmental impacts over the life cycle ('environmental footprint')" (European Commission 2011b). The EC has therefore developed and tested methods for both product and organisation environmental footprinting (European Commission 2013a, b). This article focuses on important methodological issues for the development of the EC method for Organisation Environmental Footprint (EC OEF) studies (European Commission 2013b).

To date, a variety of methods and standards (or drafts thereof) have emerged for quantifying the environmental performance of organisations (WRI and WBCSD 2004; GRI 2006; ISO 14064-1 2006; ISO 14064-3 2006; ISO/WD TR 14069 working draft 2 2010; ADEME 2007; DEFRA 2009; CDP 2010a, b; WRI and WBCSD 2011; European Commission-JRC-IES 2010a, b, c, d; 2011a; 2013a, b). These methods vary in terms of their scope, requirements, and the probable consistency of analytical outcomes. In general, current guidance for organisation-level environmental accountancy is less developed and prescriptive than for productlevel studies. In light of the influential role of organisation-level decision making in shaping environmental outcomes, further development and standardisation of supporting methods for organisation environmental footprint studies is desirable.

Clearly, the EC OEF method should, to the extent possible, align with existing methodological norms. This is desirable not only in terms of adopting and reinforcing existing best methodological practices, which have evolved thanks to the on-going efforts of diverse stakeholders, but also to reduce the potential for increasing the burdens (financial, time, and human resource) associated with environmental footprinting - in particular for organisations already using one or more existing methods. However, it is also acknowledged that the provision of a common methodology that is sufficiently robust to support the spectrum of potential applications constitutes the primary guiding principle of this undertaking. Hence, it was recognised from the outset that it may be necessary to diverge from/go beyond existing methods as appropriate to this objective (Fig. 1).

In order to support a broad range of potential applications, four core criteria were identified as essential to a common EC OEF method. Taken together, these four criteria are intended to ensure achievement of consistently higher quality analyses than might otherwise be achievable based on existing, competing methodological guidance documents.

Specifically, these criteria are that the method should:

1. cover the entire life cycle along the supply chain: This requires taking into consideration all relevant environmental interactions associated with a good, service,

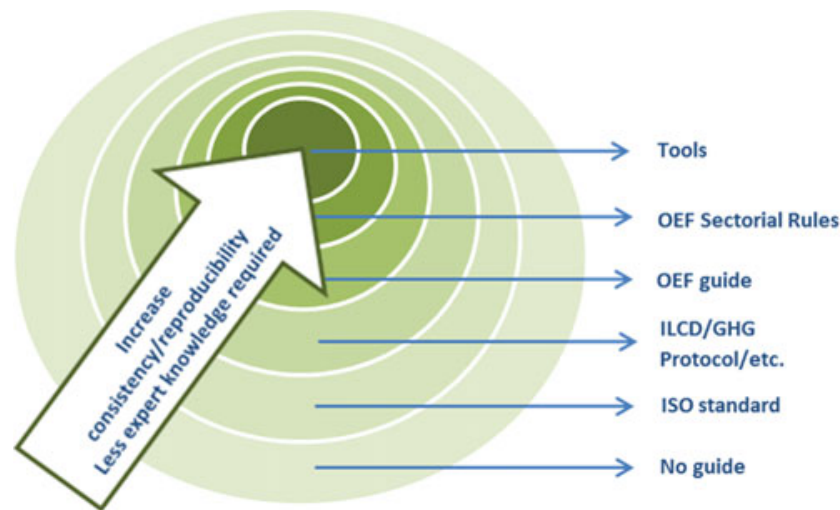

Fig. 1 Situating standards and methods for organisation environmental footprinting along the continuum from flexibility to prescriptiveness/ reproducibility

activity, or entity from a supply chain perspective, i.e. from the level of primary resource extraction through processing, distribution, use, and eventual disposal or reuse stages. Such an approach is essential to effective environmental management because important effects may occur "upstream" or "downstream" from a given focal point, and hence may not be immediately evident. Also, this approach helps to ensure that organisations do not benefit from "outsourcing" steps/life cycle stages which are linked to high environmental burdens.

2. provide for multi-criteria environmental assessments, i.e. incorporate all relevant environmental performance criteria as opposed to only a single dimension such as greenhouse gas (GHG) emissions. This is essential to making transparent any potential trade-offs between different types of environmental impacts associated with specific management decisions to help avoid unintended shifting of burdens.

3. increase reproducibility and comparability by emphasising prescriptiveness over flexibility to ensure that the method is applied consistently, within and across studies. This is necessary to produce a method that can be useful for application in the context of various possible policy instruments at EU level.

4. maximise the physical representativeness of the study outcomes, i.e. ensure that the study accurately depicts the flows of material and energy resources and emissions associated with the supply chains directly linked to the organisation's activities in order to provide the most realistic basis possible for improved environmental management. This requires that the modelling parameters be defined in concrete, physical terms. Efforts were therefore made to preclude the use of (for example) financial information wherever use of such information introduces distorting biases into the model, such that analytical 
outcomes reflect these other considerations rather than providing for physically representative results.

The development of the EC OEF method began with a review of six existing methods that are used to measure the environmental performance of organisations. The intention of this review was to (a) provide a detailed analysis of the methods in order to identify commonalities and differences, (b) identify key areas where more prescriptive and/or different requirements are necessary in order to satisfy the four core criteria, (c) provide justifications for deviations from common practice with reference to these criteria, and (d) arrive at a recommended suite of methodological requirements in these key areas for a common EC OEF method. Here, we present the results of this analysis and the key methodological requirements (along with supporting rationales) that were subsequently developed for the EC OEF method (European Commission 2013b). For an elaborated treatment of the methods for handling End-of-Life (EoL) problems in OEF studies, see Allacker et al. (2013).

\section{Methods: process of formulating the requirements for the EC OEF method}

The formulation of the key methodological recommendations for the EC OEF method proceeded according to the following steps. First, the requirements/recommendations for a subset of key methodological decision points provided by each method were screened in order to establish commonalities and differences (European Commission-JRC-IES 2011c). The available options represented for each methodological decision point were subsequently assessed against the four previously described core criteria that we consider to be essential for a common EC OEF method. In cases where (a) the reviewed methods were in general agreement and (b) the required methodological choice supported satisfaction of the four criteria, then the identified choice was to be preferentially adopted for the recommended EC OEF method. Wherever the reviewed methods presented divergent requirements, these were to be screened against the four criteria in order to identify preferred options. In cases where none of the available options satisfied the four criteria, an alternative methodological specification was to be formulated along with a supporting rationale.

Prior to the finalization of the methodological requirements, a draft EC OEF method guidance document was created (European Commission-JRC-IES 2011b). Training in use of this guidance document was provided to ten representative companies, which conducted pilot studies of their organisations using the draft EC OEF method. A 2-day invited stakeholder consultation was also hosted, along with a public commenting period. The outputs of these activities informed revisions of the methodological requirements in order to arrive at the final version of the supporting EC OEF method guidance document (European CommissionJRC-IEE 2012). These activities are not further discussed here, other than in support of the conclusions of our analysis.

2.1 Organisation environmental footprint methods considered

Six organisation environmental footprint methods were selected for analysis. A short description of each method follows.

2.1.1 ISO 14064:2006 Greenhouse gases-Part 1 and 3 (ISO 2006b, c) and ISO/WD TR 14069 working draft 2 (2010): Greenhouse gases (GHG) - Quantification and reporting of GHG emissions for organizations (Carbon footprint of organization) - Guidance for the application of ISO 14064-1

ISO 14064-1:2006 specifies principles and requirements at the organisation level for quantification and reporting of GHG emissions and removals.

ISO 14064-3:2006 specifies principles and requirements and provides guidance for those conducting or managing the validation and/or verification of GHG assertions. It can be applied for organisational or GHG project quantification, including GHG quantification, monitoring, and reporting carried out in accordance with ISO 14064-1 or ISO 14064-2.

ISO/WD TR 14069 working draft 2 (2010) provides guidance in the application of ISO 14064.

\subsubsection{Greenhouse Gas Protocol Corporate Accounting and Reporting Standard and Greenhouse Gas Protocol Corporate Value Chain (Scope 3) Accounting and Reporting Standard (WRI and WBCSD 2004, 2011)}

The Greenhouse Gas (GHG) Protocol Corporate Accounting and Reporting Standard (herein, GHG Protocol Corporate Standard) was jointly developed by the World Resources Institute and the World Business Council on Sustainable Development (WBCSD). The GHG Protocol Corporate Standard (2004) provides standards and guidance for companies and other types of organisations preparing a GHG emissions inventory. It accommodates accounting and reporting of the six GHGs covered by the Kyoto Protocol. It was first published in 2001, followed by a revised edition in 2004. According to Ascui and Lovell (2011), the GHG Protocol Corporate Accounting and Reporting Standard constitutes the current de facto standard for carbon accounting for organisations. It is recommended as the basis for carbon accounting under both the Global Reporting Initiative (GRI 2006) and the Carbon Disclosure Project (CDP 2010a).

The Greenhouse Gas Protocol Corporate Value Chain (Scope 3) Accounting and Reporting Standard (herein referred to as the GHG Protocol Scope 3 Standard), published in 2011, is a supplement to, and intended to be used in 
conjunction with, the GHG Protocol Corporate Standard. For this reason, we consider the two in tandem for the purpose of the current analysis. The GHG Protocol Scope 3 Standard provides additional requirements and guidelines on life cycle accounting and reporting of corporate "Scope 3" (value chain) emissions.

The "3 Scopes" distinction was developed by the GHG Protocol Corporate Accounting and Reporting Standard for the purpose of defining boundaries in organisation-level GHG accounting. According to this concept, Scope 1 refers to "direct emissions" (i.e. direct emissions from sources that are owned or controlled by the reporting company). Scopes 2 and 3 refer to "indirect emissions" (i.e. emissions that are related to the activities of the reporting company but occur at sources owned or controlled by another company). Scope 2 includes the emissions from the generation of purchased or acquired electricity, steam, heating, or cooling consumed by the reporting company. All other indirect emissions are included in Scope 3 (WRI and WBCSD 2011). Hence, a Scope 1 analysis is effectively a "gate-to-gate" quantification of GHG emissions, whereas a Scope 2 analysis includes, in addition, a limited subset of "upstream" emissions. Only a Scope 3 analysis provides a system boundary that can be considered to be life cycle-based in terms of supply chain inclusiveness. Accounting for Scope 3 emissions is optional when using the GHG Protocol Corporate Standard, but mandatory when the GHG Protocol Scope 3 standard is used.

\subsubsection{Bilan Carbone (version 5.0; ADEME 2007)}

Bilan Carbone (version 5.0) is a GHG accounting method developed by the French Agency for the Environment and Energy Management (ADEME). It consists of a guidance document together with supporting calculation spreadsheets (ADEME 2007) for use by organisations (including both private and public sector bodies and territorial entities). This method can be used for reporting in the framework of ISO 14064, the GHG Protocol, or the Carbon Disclosure Project. The guidance provided is more comprehensive than most other corporate GHG accounting methods. Emphasis is placed on physical realism. All GHGs (rather than only the six Kyoto Protocol gases) are considered.

\subsubsection{DEFRA 'Guidance on how to measure and report your greenhouse gas emissions' (DEFRA 2009)}

The UK 'Guidance on how to measure and report your greenhouse gas emissions' was developed by the Department of Environment, Food and Rural Affairs (DEFRA). It is largely based on the GHG Protocol Corporate Standard. It is intended to support organisations in reporting their GHG emissions, either voluntarily or to meet reporting requirements, under the Companies Act. The guidance sets minimum recommendations for what companies should report, including all scope 1 and 2 emissions within the chosen organisational boundary, and an intensity ratio. Reporting significant scope 3 emissions is discretionary but encouraged. The guidance is accompanied by annually updated emissions conversion factors and a calculation tool. The guidance also provides additional information to help companies report emissions reductions, set reduction targets, and recalculate their base year. A separate version of the guidance is available for small- and medium-sized enterprises (SMEs).

\subsubsection{CDP Water Disclosure (CDP 2010b)}

The CDP (Carbon Disclosure Project) Water Disclosure program collects and distributes information on water-related business risks and opportunities for the purpose of informing institutional investors (CDP 2010b). Areas covered by CDP Water include (1) water management and governance; (2) risks and opportunities related to water, both in a company's own operations and in their supply chains; and (3) water accounting metrics. The CDP Water disclosure guidance incorporates definitions and approaches established by the GHG Protocol and the Global Reporting Initiative (GRI) for the purpose of quantifying water usage.

\subsubsection{Global Reporting Initiative (version 3.0; GRI 2006)}

The GRI Reporting Framework establishes economic, environmental, and social sustainability performance indicators for organisations. Developed through a consensus-seeking, multi-stakeholder process by participants from global business, civil society, labour, academic and professional institutions, GRI's framework is intended to support both accountancy and reporting.

\section{Results and discussion}

The summary of our analysis and the final key EC OEF requirements are presented in Tables 1, 2, 3, 4, 5, 6, 7, 8, 9, 10, 11, and 12 and discussed below. This does not present the exhaustive suite of methodological considerations that are addressed in the EC OEF method, but rather focuses on what we consider to be the most important issues. In general, these are methodological decision points that will critically determine the extent to which the methods satisfy the four necessary criteria, and where it appears that current norms may be insufficient. While in some cases the requirements prescribed in the EC OEF method align with several or even most of the reviewed methods (for example, most (but not all) of the reviewed methods ascribe to a life cycle approach), agreement across all of the methods reviewed was not observed for any of the following methodological decision points considered. 
Table 1 Recommendation based on the comparison of the six methods regarding the issue: coverage of the entire life cycle

\begin{tabular}{lll}
\hline Methodology & Requirement & Core criteria not satisfied $^{\mathrm{a}}$ \\
\hline ISO 14064 and ISO WD/TR 14069, DEFRA & For Scope 3 (optional) & 1 , if not Scope 3 \\
GHG Protocol Corporate and Scope 3 Standards & For Scope 3 & 1, if not using Scope 3 standard \\
Bilan Carbone & Yes & 1 \\
CDP Water Disclosure, GRI & No & \\
EC OEF recommendation & Yes (mandatory, unless specifically justified) & \\
\hline
\end{tabular}

a $1=$ "cover the entire life cycle"

\subsection{Flexibility versus prescriptiveness}

As a first observation, it was noted that the reviewed guidance documents for measuring the environmental performance of organisations appear to be considerably less prescriptive than comparable guidance documents for product-level assessments. This may be because product-level standards tend to take the ISO 14044 standard (ISO 2006a) as a starting point, whereas no similar, internationally accepted methodological norm for organisation-level life cycle assessment is available (ISO is currently developing ISO/NP TS 14072 Life cycle assessment-Additional requirements and guidelines for organizations). It may also reflect that life cycle-based organisation environmental footprinting is potentially more challenging than is product-level footprinting - in particular for large organisations producing multiple products and/or operating in multiple sectors.

In general, it appears that most existing methods are intended to support internal use or high-level corporate sustainability reporting rather than to provide a robust and detailed description of environmental performance at an organisational level. Hence, such guidance documents tend to provide recommendations rather than requirements, and often present a range of methodological alternatives instead of fixing a single norm for a given decision point.

To ensure that EC OEF studies are conducted in a consistent and reproducible manner (third core criterion), the EC OEF method must necessarily be highly prescriptive. This sentiment is echoed by Moneva et al. (2006), who observe that "the lack of ... restrictive conditions such as a clear definition of the entity boundaries, the development/requirement of integrated indicators or the attachment of an independent verification statement leads to a relaxation of the basic aim, that is, sustainability."

\subsection{Covering the entire life cycle}

Few of the reviewed organisation environmental footprint methods require coverage of the entire life cycle with respect to inclusion of supply chain stages associated with organisation activities. Notable exceptions are the Bilan Carbone method and the GHG Protocol Scope 3 Standard. Because the first criterion for a common EC OEF method is that it adopts an approach covering the entire life cycle, such a common method must necessarily diverge from most of the reviewed standards in this respect. However, many of the reviewed methods do provide for optional "Scope 3" life cycle modelling, and hence are complementary to the EC OEF method in this respect when Scope 3-level analyses are undertaken (Table 1).

\subsection{Providing a comprehensive, multi-criteria assessment}

Of the reviewed methods, only GRI stipulates that a broad suite of relevant environmental impacts must be considered. All other methods refer to single impact categories only. To satisfy criterion 2, the EC OEF method therefore necessarily diverges from the reviewed methods in this respect (Table 2;

Table 2 Recommendation based on the comparison of the six methods regarding the issue: multi-criteria assessment

\begin{tabular}{|c|c|c|}
\hline Methodology & Requirement & $\begin{array}{l}\text { Core criteria } \\
\text { not satisfied }^{\mathrm{a}}\end{array}$ \\
\hline $\begin{array}{l}\text { ISO } 14064 \text { and ISO WD/TR 14069, GHG Protocol } \\
\text { Corporate and Scope } 3 \text { Standards, Bilan Carbone, DEFRA }\end{array}$ & GHG focus & 2 \\
\hline CDP Water Disclosure & Focus on water issues & 2 \\
\hline GRI & Comprehensive multi-criteria assessment & \\
\hline EC OEF recommendation & $\begin{array}{l}\text { Comprehensive multi-criteria assessment - any exclusion of } \\
\text { required impact categories must be justified and } \\
\text { supported by appropriate documents (subject to review) }\end{array}$ & \\
\hline
\end{tabular}

a $2=$ "provide for multi-criteria assessments" 
Table 3 Recommendation based on the comparison of the six methods regarding the issue: functional unit and reference flow

\begin{tabular}{|c|c|c|}
\hline Methodology & Requirement & Core criteria not satisfied ${ }^{\mathrm{a}}$ \\
\hline $\begin{array}{l}\text { ISO } 14064 \text { and ISO WD/TR 14069, GHG Protocol } \\
\text { Corporate and Scope } 3 \text { Standards, Bilan Carbone, } \\
\text { DEFRA, CDP Water Disclosure, GRI }\end{array}$ & $\begin{array}{l}\text { Does not use concepts of functional unit } \\
\text { and reference flow }\end{array}$ & 3,4 \\
\hline EC OEF Recommendation & $\begin{array}{l}\text { Uses concept of functional unit (organisation as } \\
\text { goods/service provider) and reference flow } \\
\text { (Product Portfolio=the sum of goods/services } \\
\text { provided by the organisation over the reporting interval) }\end{array}$ & \\
\hline
\end{tabular}

a $3=$ "increase reproducibility and comparability", 4="maximise the physical representativeness of the study outcomes"

see Section 3.8 for the required suite of environmental footprint impact assessment models for EC OEF studies).

\subsection{Defining the functional unit (unit of analysis)} and reference flow

In product-oriented environmental footprinting analyses, it is standard to define a functional unit and a reference flow. The functional unit, also termed the unit of analysis (as adopted for the EC OEF method), is a quantitative description of the performance of the product system in terms of "what, how much, how well, and for how long" (EC JRC IES 2010a). The reference flow is the amount of product necessary to provide the defined functional unit. Taken together, these serve as the basis for precisely specifying the system to be analysed. Although not employed in any of the reviewed methods (Table 3), these concepts are similarly useful and, indeed, necessary for life cycle-based organisation environmental footprint accountancy. This is because (if appropriately implemented) they firmly ground the analysis in concrete, physical relationships that connect upstream, organisationlevel, and downstream processes in a coherent, internally consistent manner. Ensuring such internal consistency is essential for arriving at realistic, physically representative organisation environmental footprint studies. For this reason, the
EC OEF method departs from common practice (as per the reviewed guidance documents) by incorporating the concepts of functional unit and reference flow as central elements of organisation environmental footprint studies.

The unit of analysis (functional unit) for an OEF study could, however, potentially be defined in several ways, some of which are incompatible with the defined criteria for the EC OEF method. For example, the unit of analysis might be defined as simply "the organisation within its organisational (site) boundaries". Here, only a limited subset of "what" considerations are accommodated, while the "how much, how well, and for how long" dimensions are ignored. In this case, the calculated footprint would refer only to the infrastructure of the organisation and would not include site-level activities nor the goods/services provided. Because this approach is not life cycle-based, nor does it allow for a realistic, physically representative OEF, it is insufficient according to our first and fourth criteria.

A second option could be to define the unit of analysis as the organisation, including organisational activities, occurring within the site boundaries during a 1-year time interval. This approach accommodates a subset of both the quantitative and temporal dimensions. Similar to a "Scope 1" analysis, as commonly defined in the context of the reviewed GHG accounting methods, such an approach would effectively limit

Table 4 Recommendation based on the comparison of the six methods regarding the issue: organisation boundaries

\begin{tabular}{lll}
\hline Methodology & Requirement & Core criteria not satisfied $^{\text {a }}$ \\
\hline $\begin{array}{l}\text { ISO 14064 and ISO WD/TR 14069, GHG Protocol } \\
\text { Corporate and Scope 3 Standards, Bilan Carbone, }\end{array}$ & $\begin{array}{l}\text { Choice of equity share, financial control, or } \\
\text { operational control approach }\end{array}$ & $1,3,4$ \\
DEFRA, CDP Water Disclosure, GRI & $\begin{array}{l}\text { Financial/operational control and ability to } \\
\text { exert significant influence } \\
\text { GRI }\end{array}$ & $\begin{array}{l}\text { Control approach-includes all processes/ } \\
\text { activities under the financial and/or } \\
\text { EC OEF Recommendation }\end{array}$ \\
$\begin{array}{l}\text { operational control of the organisation } \\
\text { that are related to the provision of the }\end{array}$ \\
Product Portfolio
\end{tabular}

\footnotetext{
${ }^{a} 1=$ "cover the entire life cycle", $3=$ "increase reproducibility and comparability", $4=$ "maximise the physical representativeness of the study outcomes"
} 
Table 5 Recommendation based on the comparison of the six methods regarding the issue: Organisation Environmental Footprint boundaries

\begin{tabular}{|c|c|c|}
\hline Methodology & Requirement & Core criteria not satisfied ${ }^{\mathrm{a}}$ \\
\hline ISO 14064 and ISO WD/TR 14069, DEFRA & Scope 1,2 required, Scope 3 optional & $1,3,4$ \\
\hline GHG Protocol Corporate and Scope 3 Standards & $\begin{array}{l}\text { Scope 1, } 2 \text { required in Corporate Standard but } 3 \\
\text { optional unless following Scope } 3 \text { Standard }\end{array}$ & 1, 3, 4 unless following Scope 3 Standard \\
\hline Bilan Carbone & All physical flows related to the organisation & 1,3 \\
\hline CDP Water Disclosure & Not specified & $1,3,4$ \\
\hline GRI & $\begin{array}{l}\text { Account for impacts from all activities over which } \\
\text { the company has control or significant influence. }\end{array}$ & $1,3,4$ \\
\hline EC OEF Recommendation & $\begin{array}{l}\text { Account for all direct and/or indirectly attributable } \\
\text { activities occurring along the supply chains } \\
\text { associated with Organisation's Product } \\
\text { Portfolio. This shall include, at a minimum, } \\
\text { site-level (direct) and upstream (indirect) } \\
\text { activities. Explicit justification shall be provided } \\
\text { if downstream (indirect) activities are excluded. }\end{array}$ & \\
\hline
\end{tabular}

a $1=$ "cover the entire life-cycle", $3=$ "increase reproducibility and comparability", $4=$ "maximise the physical representativeness of the study outcomes"

the assessment to a gate-to-gate analysis instead of a cradle-tograve analysis, which is likewise unacceptable in light of the first and fourth core criteria.

In order to satisfy these criteria using the concepts of functional unit and reference flow, our analysis points towards the usefulness of understanding the function of the organisation to be primarily the provision of goods and/or services (i.e. products). In this way, the defined unit of analysis (the organisation and, for the purpose of downstream modelling, the functions of its products) provides the necessary basis for linking upstream, site-level, and downstream processes. Indeed, downstream modelling for organisations only becomes intelligible at the product level. Accordingly, the third (required) possible definition for the unit of analysis is the organisation, as product provider (i.e. taking into account the life cycle impacts of the products provided) over a 1-year time interval. On this basis, the reference flow for the analysis (what we term the "Product Portfolio") is the total amount of products provided by the organisation over the reporting interval. This definition provides space for accommodating all of the necessary components of the functional unit (i.e. what, how much, how well, and for how long), and also satisfies all four of the necessary criteria.

\subsection{Organisation boundaries}

As defined by ISO 14044 (ISO 2006a), the system boundaries for life cycle assessment are " $a$ set of criteria specifying which unit processes are part of a product system and thus determine which processes shall be included within the LCA". In the context of an OEF study, two levels of system boundary definition are necessary. These are, first, the organisation boundaries and, second, the life cycle (supply chain) boundaries. We refer to the latter as "Organisation Environmental Footprint boundaries". This section focuses on the definition of the organisation boundaries, while the next (3.6) focuses on the Organisation Environmental Footprint boundaries.

Defining organisation boundaries requires the identification of processes/activities directly attributable to the organisation (as a unit of analysis) versus indirect processes/ activities that are linked to organisation activities either upstream or downstream along the supply chain (i.e. indirectly

Table 6 Recommendation based on the comparison of the six methods regarding the issue: cut-off criteria

\begin{tabular}{llc}
\hline Methodology & Requirement & $\begin{array}{c}\text { Core criteria not } \\
\text { satisfied }^{\mathrm{a}}\end{array}$ \\
\hline ISO 14064 and ISO WD/TR 14069 & Based on materiality, feasibility and cost effectiveness & 3 \\
$\begin{array}{l}\text { GHG Protocol Corporate and Scope 3 Standards, } \\
\text { Bilan Carbone, DEFRA }\end{array}$ & Discouraged & 3 \\
CDP Water Disclosure & Permissible where data is lacking & 3 \\
GRI & Based on control/influence/significance \\
EC OEF Recommendation & Cut-off criteria shall not be employed & 3 \\
\hline
\end{tabular}

a $3=$ "increase reproducibility and comparability" 
Table 7 Recommendation based on the comparison of the six methods regarding the issue: Environmental Footprint impact categories and models

\begin{tabular}{|c|c|c|}
\hline Methodology & Requirement & $\begin{array}{l}\text { Core criteria } \\
\text { not satisfied }^{\mathrm{a}}\end{array}$ \\
\hline $\begin{array}{l}\text { ISO } 14064 \text { and ISO WD/TR } 14069 \text {, } \\
\text { GHG Protocol Corporate and Scope } 3 \\
\text { Standards, DEFRA }\end{array}$ & GHG emissions & 2,4 \\
\hline CDP Water Disclosure & Water use & 2 \\
\hline GRI & All relevant environmental, social, and economic impacts & 3 \\
\hline EC OEF Recommendation & $\begin{array}{l}12 \text { default mid-point environmental footprint impact categories } \\
\text { and specified impact assessment models with impact indicators. } \\
\text { The impact categories are: climate change, ozone depletion, } \\
\text { eco-toxicity-freshwater, human toxicity-cancer effects, human } \\
\text { toxicity-non-cancer effects, particulate matter/respiratory inorganics, } \\
\text { ionising radiation-human health effects, photochemical ozone } \\
\text { formation, acidification, eutrophication-terrestrial, eutrophication-- } \\
\text { aquatic, resource depletion-water, resource depletion-fossil, and land use. } \\
\text { Any exclusion shall be explicitly justified and its influence on the final } \\
\text { results discussed. Such exclusions are subject to review. }\end{array}$ & \\
\hline
\end{tabular}
a $2=$ "provide for multi-criteria environmental assessments", 4="maximise the physical representativeness of the study outcomes", $3=$ "increase
reproducibility and comparability"

attributable activities). Moneva et al. (2006) observe that "the elaboration of specified guidelines defining the reporting entity boundaries is a complex challenge and has become an absolute necessity". We agree that providing a consistent basis for defining organisation boundaries is necessary to satisfy the core criteria for a common EC OEF method.

Most of the reviewed guidance documents for organisation environmental footprint studies differentiate between three approaches to defining organisation boundaries (Table 4). First is the equity share approach, where organisation boundaries encompass all activities in which there is an ownership share. Responsibility for associated environmental burdens is subsequently assigned in direct proportion to the ownership share in the activities of concern. Second is the financial control approach, where organisations include within their defined boundaries only those activities over which they have financial control. Third is the operational control approach, where only those activities over which an organisation has operational control are included in the defined organisation boundaries. For further details on these approaches see, for example, WRI and WBCSD (2004). Clearly, in order to achieve increased comparability and reproducibility (criterion 3), the EC OEF method must necessarily deviate from existing guidance by specifying a single basis for defining organisation boundaries.

As EC OEF studies will be, a priori, life cycle-based (Criterion 1), meaning that all activities in the supply chains linked to organisation activities need be accounted for, there is a certain degree of arbitrariness as to how/where organisation boundaries are drawn. The same activities will be included in an EC OEF study as in a study using, for example, the GHG Protocol Scope 3 Standard or similar methods. However, taking into account criteria 3 and 4, we identify several reasons in support of a single, preferred approach.

First, we identify the "control" approach as preferable to the "equity share" approach. As explicitly recognised in existing guidance documents such as ISO WD/TR 14069 and the GHG Protocol Corporate Accounting and Reporting Standard (WRI and WBCSD 2004), the equity share approach is better suited to financial risk management, whereas the

Table 8 Recommendation based on the comparison of the six methods regarding the issue: specific data

\begin{tabular}{lll}
\hline Methodology & Requirement & $\begin{array}{c}\text { Core criteria not } \\
\text { satisfied }\end{array}$ \\
\hline $\begin{array}{l}\text { ISO } 14064 \text { and ISO WD/TR 14069, } \\
\text { Bilan Carbone, DEFRA }\end{array}$ & Required for activities within the organisation boundary. & 3 (for 14069 only) \\
$\begin{array}{l}\text { GHG Protocol Corporate and Scope } \\
3 \text { Standards }\end{array}$ & $\begin{array}{l}\text { No requirement. Choice of primary versus secondary data } \\
\text { should reflect study goals }\end{array}$ \\
$\begin{array}{l}\text { CDP Water Disclosure, GRI } \\
\text { EC OEF Recommendation }\end{array}$ & $\begin{array}{l}\text { Required for activities within the organisation boundary, } \\
\text { except where secondary data are more representative. }\end{array}$ \\
\hline
\end{tabular}

a $3=$ "increase reproducibility and comparability" 
Table 9 Recommendation based on the comparison of the six methods regarding the issue: generic data

\begin{tabular}{|c|c|c|}
\hline Methodology & Requirement & Core criteria not satisfied ${ }^{\mathrm{a}}$ \\
\hline ISO 14064 and ISO WD/TR 14069 & $\begin{array}{l}\text { Should be derived from a recognised source and } \\
\text { be current and appropriate }\end{array}$ & \\
\hline $\begin{array}{l}\text { GHG Protocol Corporate and } \\
\text { Scope } 3 \text { Standards }\end{array}$ & $\begin{array}{l}\text { Provides description of secondary data for each } \\
\text { category in scope } 3 \text {, and lists preferred sources }\end{array}$ & 3 \\
\hline Bilan Carbone & $\begin{array}{l}\text { Provides emission factors and average activity } \\
\text { data. Other secondary data should be sourced } \\
\text { from the European Life Cycle Data (ELCD) } \\
\text { platform and peer-reviewed data }\end{array}$ & \\
\hline DEFRA & $\begin{array}{l}\text { Provides emission factors (more site specific } \\
\text { data should be used if available) as well as } \\
\text { sources of emissions factors }\end{array}$ & \\
\hline CDP Water Disclosure, GRI & Not specified & 3,4 \\
\hline EC OEF Recommendation & $\begin{array}{l}\text { All generic data shall fulfil the data quality } \\
\text { requirements specified. } \\
\text { When available, sector-specific generic data } \\
\text { shall be used instead of multi-sector generic } \\
\text { data. Preferred sources for generic data are: } \\
\text { OEF and PEF studies, ILCD, ELCD. If not } \\
\text { available, sources should preferably be: } \\
\text { international governmental organisations, } \\
\text { national LCI database projects, third party } \\
\text { LCI databases, peer-reviewed literature. }\end{array}$ & $\begin{array}{l}\text { (3 may not always be fully satisfied } \\
\text { because different data sets may } \\
\text { comply with quality criteria) }\end{array}$ \\
\hline
\end{tabular}

a $3=$ "increase reproducibility and comparability", 4="maximise the physical representativeness of the study outcomes"

control approach is better suited to environmental performance measurement and management. This is because there is greater potential to make management changes in response to insights derived from environmental footprint studies where the organisation has direct influence over the facilities in question - whether via financial or operational control. This is a key intended application of EC OEF studies. More important, however, is that the equity share approach requires modelling all facilities/activities/processes in which the organisation has ownership share, then subsequently attributes burdens in proportion to ownership share. It therefore conflicts with criterion 4 because the attribution of burdens is based on financial considerations rather than on logical, physical cause-effect considerations. In contrast, the control approach defines boundaries at the facility/activity/process level, and allows that burdens be attributed on a physical rather than a financial basis. Hence, the latter allows for constructing an internally consistent life cycle model that quantifies and attributes burdens in direct relation to the material and energy flows linking upstream, organisation-level, and downstream activities, whereas the former does not.

Second, we identify an inclusive interpretation of the control approach (i.e. defining organisation boundaries taking into account both financial and operational control) as conducive to satisfying the fourth core criterion because it ensures that all processes/activities for which the organisation may be able to

Table 10 Recommendation based on the comparison of the six methods regarding the issue: allocation (general)

\begin{tabular}{|c|c|c|}
\hline Methodology & Requirement & $\begin{array}{l}\text { Core criteria } \\
\text { not satisfied }\end{array}$ \\
\hline $\begin{array}{l}\text { ISO } 14064 \text { and ISO WD/TR 14069, } \\
\text { DEFRA, CDP Water Disclosure, GRI }\end{array}$ & No allocation hierarchy specified & 3,4 \\
\hline $\begin{array}{l}\text { GHG Protocol Corporate and Scope } 3 \\
\text { Standards }\end{array}$ & Allocation hierarchy follows ISO 14044 , but does not refer to system expansion & 3,4 \\
\hline Bilan Carbone & Allocation hierarchy follows ISO 14044, but prohibits use of economic allocation & 3 \\
\hline EC OEF Recommendation & $\begin{array}{l}\text { (1) subdivision or system expansion; (2) allocation based on a relevant underlying } \\
\text { physical relationship (including direct substitution or some other relevant } \\
\text { underlying physical relationship); (3) allocation based on some other relationship } \\
\text { (including indirect substitution or some other relevant underlying relationship). }\end{array}$ & \\
\hline
\end{tabular}

a $3=$ "increase reproducibility and comparability", 4="maximise the physical representativeness of the study outcomes" 
Table 11 Recommendation based on the comparison of the six methods regarding the issue: EoL allocation

\begin{tabular}{|c|c|c|}
\hline Methodology & Requirement & Core criteria not satisfied ${ }^{a}$ \\
\hline $\begin{array}{l}\text { ISO } 14064 \text { and ISO WD/TR 14069, DEFRA, } \\
\text { CDP Water Disclosure, GRI }\end{array}$ & None specified & 3,4 \\
\hline GHG Protocol Corporate and Scope 3 Standards & $\begin{array}{l}\text { Emissions from recycling to be reported } \\
\text { separately for recycled content and } \\
\text { recyclable material at EoL. Companies } \\
\text { should not report negative or avoided } \\
\text { emissions associated with recycling in } \\
\text { the normal reporting categories. Any } \\
\text { claims of avoided emissions associated } \\
\text { with recycling may instead be reported } \\
\text { separately. }\end{array}$ & 4 \\
\hline Bilan Carbone & $\begin{array}{l}\text { Stock method for closed loop, avoided } \\
\text { impact method for open loop }\end{array}$ & 3,4 \\
\hline EC OEF Recommendation & $\begin{array}{l}\text { Adapted 50/50 approach, single equation } \\
\text { for different EoL scenarios }\end{array}$ & $\begin{array}{l}4 \text { is not achieved at the product } \\
\text { level, but is satisfied at the } \\
\text { system level }\end{array}$ \\
\hline
\end{tabular}

a $3=$ "increase reproducibility and comparability", 4="maximise the physical representativeness of the study outcomes"

leverage system specific (i.e. primary) data are included within the organisation boundary. This is essential to ensuring maximally representative models that will support differentiation in the context of possible applications. Generic data will then only be needed for the upstream and downstream processes/activities outside the organisation boundaries which are included in the Organisation Environmental Footprint boundaries. This approach also provides the least opportunity for confusion in system boundary setting and associated potential for double-counting or under-counting of activities for jointly owned or operated facilities (criterion 3 ). Because only one organisation will market a given product, the division of associated activities has an unambiguous basis. In other words, by choosing the control approach AND linking the definition of organisation boundaries to the products provided, we circumvent the ambiguities that can arise when organisation boundaries are defined using ONLY the control principle (whether financial or operational), particularly where ownership or management structures are complex.

\subsection{Organisation Environmental Footprint boundaries}

Defining organisation boundaries is useful from the perspective of describing directly versus indirectly attributable life cycle impacts associated with the activities of an organisation. However, accounting solely for the impacts of activities occurring within the defined organisation boundary does not provide a sufficient representation of an organisation's environmental impact (Moneva et al. 2006; Schaltegger and Burrit 2010). Instead, life cycle-based environmental accountancy requires an expanded definition of system boundaries that encompasses all relevant supply chain stages (Moneva et al. 2006). Among the methods reviewed, almost all define system boundaries for an analysis in terms of the " 3
Scopes" distinction, as developed by the GHG Protocol (WRI and WBCSD 2004; Table 5).

In light of the widespread adoption of the 3 Scopes distinction, there is clearly appeal in similarly employing this concept in the EC OEF method. However, we identify several compelling reasons which suggest an alternative basis for defining system boundaries is preferable for the intended purposes.

First, neither Scope 1 nor Scope 2 analyses are, by definition, life cycle-based, hence they cannot be accommodated in the EC OEF method. Second, the difference between "Scope 2" and "Scope 3" has meaning in the context of non-life cycle-based GHG accounting only, where it may be desirable to include upstream emissions related to the provision of the utilized energy carriers (for example, electricity, steam, heating and cooling energy) in addition to direct "Scope 1" emissions for a selectively expanded gateto-gate analysis. However, this is not relevant in the context of life cycle-based, multi-criteria environmental accounting studies because "Scope 2" does not provide a useful differentiation for impact categories other than GHG emissions. Finally, although it might be feasible to incorporate a modified conception of the 3 Scopes concept into the EC OEF method, it is likely that such a modified conception would bring more confusion than clarity to both GHG accounting and environmental footprinting. For these reasons, we suggest that it is more useful to employ system boundary terminology that is consistent with life cycle assessment practise rather than (contemporary) GHG accounting practice.

We hence propose adoption of the terms site-level, upstream (i.e. cradle-to-gate) and downstream (gate-to-grave) impacts as a basis for distinguishing between directly and indirectly attributable activities and impacts (Fig. 2). To fulfil the first core criterion (life cycle approach), the organisation 
Table 12 Recommendation based on the comparison of the six methods regarding the issue: data quality requirements

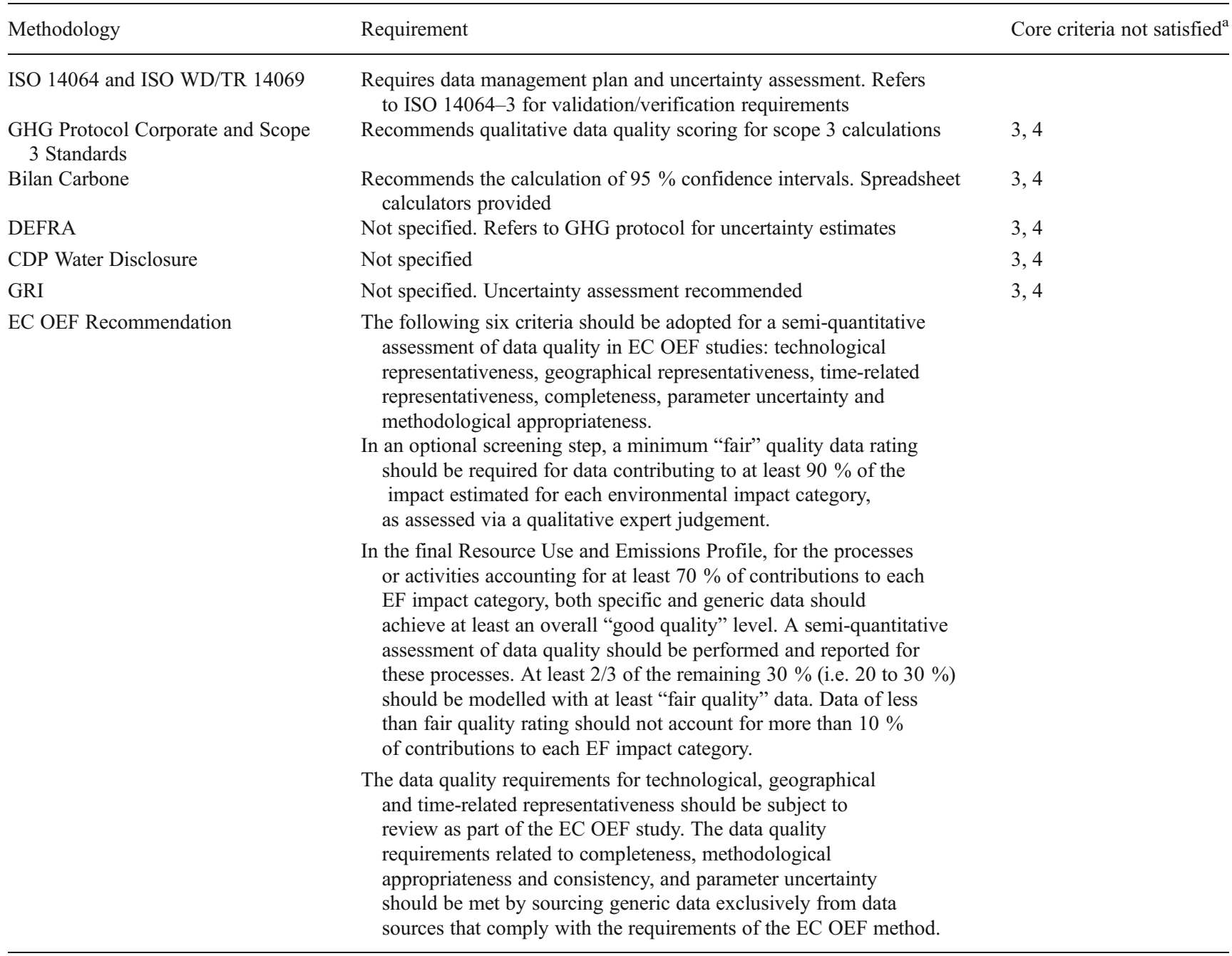

a $3=$ "increase reproducibility and comparability", 4="maximise the physical representativeness of the study outcomes"

environmental footprint boundaries in the EC OEF method should therefore be defined as including all upstream and downstream processes/activities linked to the organisation's Product Portfolio. This is similar to the GHG Protocol Scope 3 requirement.

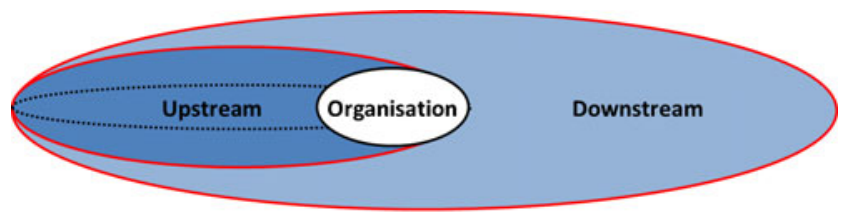

Organisation Boundary (Direct) and GHG Protocol Scope 1

GHG Protocol Scope 2 (not used in OEF studies)

Organisation EF Boundary (Indirect) and GHG Protocol Scope 3

Fig. 2 Proposed organisational and OEF boundaries, contrasted with GHG Protocol 3 Scopes boundaries
Although the EC OEF method is, a priori, life-cycle based, which requires cradle-to-grave environmental accountancy, it should be recognised that, in certain situations, such accountancy may simply not be feasible. For example, where an organisation produces intermediate products of indeterminate fate, it may simply not be possible to arrive at concrete, cradle-to-grave models. Here, the elaboration of sector-specific rules in complement to the EC OEF method will be necessary in order to precisely define use and EoL scenarios as appropriate to the sector and product category, in order to ensure comparability between studies. In the absence of such guidance, cradle-to-gate analyses may, in some circumstances, be defensible.

\subsection{Cut-off criteria}

Following Sangwon et al. (2004), "considering the complex interdependence of processes in modern economies, it would be fair to assume that in general all processes are directly or 
indirectly connected." Establishing system boundaries for analyses of OEFs in a systematic manner is therefore challenging. The appropriateness of system boundaries will ultimately be influenced by the completeness of the generic data sets used to represent processes upstream and downstream of the organisation boundary (see Section 3.13 on data quality requirements). Once such boundaries are established, it is also necessary to treat processes within the system boundaries in a similarly systematic manner. In principle, it is desirable that all activities/processes that are attributable to the analysed system within the defined system boundaries are considered in OEF studies. In practice, however, not all of these activities/ processes may be environmentally significant, nor may it be possible to acquire the data necessary to include these in the OEF model. For these reasons, cut-off criteria are often established in environmental accountancy models. They provide thresholds for inclusion of environmentally significant processes and flows, in the interest of balancing returns on effort and analytical robustness (Sangwon et al. 2004).

Among the reviewed methods, no common approach to establishing cut-off criteria was identifiable (Table 6). While some methods discourage their use, others specify, at a general level, the basis for determining cut-off criteria. None of the methods presented clearly defined cut-off criteria, nor disallowed their use.

Elsewhere in environmental accountancy, cut-off criteria are sometimes defined in terms of a proportion of energy or mass flows at the inventory level, or as a specified maximum contribution of the cut-off flows to the impact categories considered (for example, see EC JRC IES 2010a; ISO 2006a; and Sangwon et al. 2004). Because the EC OEF method does not provide recommended methods for energy or mass flow analysis, neither of these options are available as potential cut-off criteria (see subsequent section on impact assessment methods).

For the purpose of the EC OEF method, if cut-offs were to be entertained then they must necessarily be identified on a systematic basis, so as to ensure reproducibility/consistency among studies (criterion 3), and on the basis of environmental impacts in order to ensure that no environmentally relevant flows are cut off (criterion 4). The option of defining cut-off criteria based on a predetermined level of contribution to impacts appears, at first instance, feasible in the context of the EC OEF method because required impact assessment methods are specified. However, we foresee that some non-trivial, practical problems would arise due to the multi-criteria nature of the EC OEF method, which render this option unattractive. While it would seem straightforward to determine flows to be cut off on the basis of impact contributions for a single criteria assessment (for example, if only GHG emissions are considered), this will not be the case where multiple impact categories are considered. Flows will contribute differently to each impact category, meaning that different flows will need to be cut off. This would effectively require determining cut-offs for each impact category relative to the threshold, with parallel final impact assessments and interpretations in each case.

Moreover, in order to determine what may be cut off, the whole system must be first modelled in some way (be it based on a kind of screening); hence, potentially cut-off flows might as well anyway be included in some form in the analysis. In light of these considerations, it was decided that the EC OEF method will disallow the use of cut-offs. It should be noted, however, that the recommended data quality provisions effectively allow for the use of "proxy data" in place of cut-offs (see Section 3.13).

\subsection{Environmental footprint impact categories and models}

Impact categories refer to specific categories of environmental impacts considered in an environmental accountancy study. These are generally related to resource use (for example, fossil fuels and mineral ores) or emissions of environmentally problematic substances, such as GHGs or toxic chemicals. Environmental footprint impact assessment methods use models for quantifying the causal relationships between the material/energy inputs and emissions associated with organisation activities (inventoried in the Resource Use and Emissions Profile) and each environmental footprint impact category considered. Each impact category hence has an associated, stand-alone environmental footprint impact assessment model and impact indicator.

The purpose of environmental footprint impact assessment is to group and aggregate the collected inventory data (Resource Use and Emissions Profile) according to the respective contributions to each impact category. This subsequently provides the necessary basis for interpretation of the environmental footprint results relative to the goals of the footprint study (for example, identification of supply chain "hot spots" and options for improvement). The selection of environmental footprint impact categories must therefore be comprehensive in the sense that they cover all relevant environmental issues related to the activities of the organisation of interest.

All reviewed methods other than the Global Reporting Initiative provide for single criterion environmental assessments - most commonly, organisation GHG accounting but also including water footprinting (Table 7). This single-criterion approach is clearly in conflict with both the first and second core criteria for the EC OEF method. For many of the GHG accounting methods, which require accounting for the six "Kyoto" GHGs only, the fourth criterion is also compromised. GRI, in contrast, stipulates that all relevant impacts (social, economic, and environmental) should be considered. However, supporting models are provided for only a limited subset of these impacts (contradicting criterion 3 ). 
A broad suite of impact assessment categories and supporting models are available for potential use in the EC OEF method. In order to meet criteria 2 (multi-criteria assessment), 3 (reproducibility/consistency), and 4 (maximise physical representativeness), the EC OEF method must specify: (1) a required suite of relevant environmental impact categories and (2) the best available impact assessment models to employ. The International Reference Life Cycle Data System (ILCD) includes a suitable, systematic review of available methods and supporting models as a basis for its recommendations (EC JRC IES 2011a). It was therefore decided that the EC OEF method will adopt the suite of recommended mid-point methods (because of its lower level of uncertainty than endpoint methods) from the ILCD Handbook (Table 7). These must necessarily be updated over time to reflect evolving best practices in this field. For details regarding the requirement methods, models, category indicators and sources, see EC JRC IES $2011 \mathrm{a}$.

It should be noted that in many contexts, data availability may currently be insufficient to support application of all 14 of the specified impact categories. This applies, in particular, to modelling abiotic resource depletion-water, land use, and toxicity impacts.

\subsection{Specific data}

Specific data (also referred to as primary data) refer to directly measured or collected data representative of activities at a specific facility or set of facilities. These should be used for as many processes and activities as possible so as to best reflect the physical reality of organisation activities (core criterion 4). Requirements among the guidance documents reviewed regarding specific data differed widely (Table 8). ISO 14064 and ISO WD/TR 14069, Bilan Carbone, and DEFRA require specific data for activities with in the defined organisation boundary, whereas the GHG Protocol Corporate and Scope 3 Standards suggest that the choice of specific versus generic data should reflect the goals of the study. CDP Water Disclosure and GDI provide no specifications at all in this regard.

To best reflect physical reality, the collection of specific data is mandatory in the EC OEF method for all processes/activities over which the organisation has financial and/or ownership control (and, hence, access to the necessary data) and for background processes/activities where appropriate. In some exceptional cases, generic (secondary) data may be more representative for a certain activity/process within or outside of the organisation boundary. In these cases, the EC OEF method requires that any such generic data be used-for example, where supply chains are not fixed because certain inputs are sourced from multiple suppliers in response to changing market prices.

\subsection{Generic data}

Generic data (also referred to as secondary data) refer to data that are not directly collected, measured, or estimated, but rather sourced from a third-party life cycle inventory database or other source. Most often, specific data will not be available for upstream and/or downstream processes, hence generic data will be used. Requirements among the guidance documents reviewed regarding generic data differed widely (Table 9).

The EC OEF method necessarily permits use of generic data for processes/activities outside of the organisation boundary, as well as in exceptional instances where specific data are unavailable for processes/activities within the organisation boundary. In all cases, the most representative data must be sourced (core criterion 4). For example, sector-specific generic data are preferred over multi-sector generic data. To further improve consistency (core criterion 3), preferred sources for generic data are provided in the EC OEF method guidance document. All such data must fulfil the minimum stipulated data quality requirements (see Section 3.13).

\subsection{Allocation (general)}

Allocation problems arise wherever input flows and resultant emissions need to be partitioned among co-products or coprocesses. Such problems are characteristic of several distinct situations that may potentially arise in OEF studies. If a process or facility that is under the financial and/or operational control of the reporting organisation provides several products, and only a subset of these are attributable to the organisations' defined Product Portfolio, then allocation will need to be performed between these goods/services. This includes both (a) between products produced independently of each other and (b) between co-produced products. In these situations, all inputs and emissions linked to the process must be partitioned between the product of interest and the other coproducts in a principled manner in order to ensure both reproducibility/consistency (criterion 3) and physical representativeness (criterion 4). However, a variety of competing bases may be potentially applied to resolve allocation problems.

The ISO 14044 standard (ISO 2006a) provides the most widely recognised allocation hierarchy. However, there is no convergence between the reviewed methods as to a preferred allocation hierarchy (Table 10), and several depart from ISO 14044. For example, economic allocation is disallowed in the Bilan Carbone standard in order to increase physical representativeness (ADEME 2007), while system expansion is excluded from the GHG Protocol Scope 3 Standard (WRI and WBCSD 2011). Nor does the ISO hierarchy provide a sufficiently detailed basis for identifying appropriate allocation solutions. In particular, the wording of the ISO hierarchy is vague, hence allowing for alternative interpretations. A review of relevant LCA literature suggests that competing 
interpretations are the norm (Ekvall and Finnveden 2001; Guinee et al. 2002; Brander and Wylie 2011; Pelletier and Tyedmers 2011; Wardenaar et al. 2012). It was therefore deemed necessary that the EC OEF method articulate more precisely what is meant by each step in the adopted multifunctionality hierarchy (criterion 3), and also that the preferred allocation solutions be those that result in physically representative modelling outcomes (criterion 4). However, it is recognised that further prescription (i.e. fixed allocation scenarios and factors) at the level of sectorial guidance documents will be necessary in the future to further increase the extent to which both of these criteria are achieved.

Taking ISO 14044 as a starting point, the EC OEF method requires application of the allocation hierarchy presented in Table 10. Similar to ISO 14044, sub-division or system expansion represent preferred solutions to (avoiding) allocation problems. We clarify, however, that expanding the system to include the functions of the co-products refers here to the calculation and reporting of impacts at the level of all coproducts (i.e. at the system level). Subsequent steps of modelling alternative co-product supply chains and subtracting the inventories in order to isolate the inventory attributable to the co-product of interest (i.e. substitution) is explicitly excluded from this step of the hierarchy (instead, substitution is defined as two specific applications of allocation-see below). This is clearly preferable according to criterion 4 because the potential for the use of arbitrary or poorly representative substitution scenarios as preferred solutions is reduced.

Also in line with ISO 14044, the second tier of the adopted allocation hierarchy requires the identification of an underlying physical relationship as the basis for partitioning input/output flows. Here also, we provide further specification/clarification to that provided by ISO 14044 in two ways. First, we identify substitution based on a direct, empirically demonstrable relationship as a possible form of physical allocation. An example of such a substitution is when manure nitrogen is applied to agricultural land, directly substituting for an equivalent amount of the specific fertilizer nitrogen that the farmer would otherwise have applied. Here, the system is expanded to include production of the specific nitrogen fertilizer, and the animal husbandry system from which the manure is derived is credited for the substituted fertilizer production. This is distinct from assumed substitutions "at the margin" based on hypothetical market models, as is typical of much consequential LCA modelling. Secondly, we add the term "relevant" as an important and necessary descriptor for an underlying physical relationship. In this context, the relationship should be relevant either in the sense that it reflects how input flows determine the proportions of output flows, or in terms of how specific characteristics of the input flows relate to the functions provided by the co-products.

The third tier of our adopted allocation hierarchy, again similar to ISO 14044, refers to allocation based on some other relevant relationship. As the last tier of the hierarchy, these "last resort" solutions should only be applied when it is not possible to identify a tier one or tier two solution. We include here allocation based on hypothetical substitution scenarios. Another example frequently invoked in relation to this tier is economic allocation, although such a solution is in clear contradiction with criterion 4 as it typically results in model outcomes that reflect market relationships rather than physical relationships (Pelletier and Tyedmers 2011, 2012).

\subsection{End-of-Life allocation}

Dealing with multi-functionality of products is particularly challenging when recycling or energy recovery of one (or more) of these products is involved. Several approaches exist and are currently applied for solving multi-functionality problems at EoL. Only a few of the reviewed methods provide recommendations in this respect, and these are varied (Table 11).

A detailed description of and comparison with the approaches analysed for the development of the EoL formula for the EC OEF method is provided in Allacker et al. (2013). Briefly summarised, three common approaches can be distinguished:

The "recyclability substitution" approach (also referred to as "EoL recycling", "0-100", "avoided burden", "avoided impact" or "closed-loop approximation" approach) is based on the assumption that the recycled material retains the same inherent properties as the original virgin material input to the life cycle. Burdens are only assigned to the recycling at EoL and credits are given, in proportion to the recycling rate, for avoided production of virgin materials. Recycled inputs entering the facility are assigned burdens commensurate with the production of an equivalent amount of virgin material. The content of recycled material in the product itself is thus not considered (Frischknecht 2010; European Commission 2010a; ADEME 2007). This approach constitutes, in essence, a form of "substitution".

The "recycled content" approach (also referred to as the "cut-off", "100-0" or "stock" approach) considers the recycled content of a product only, and does not assign any impacts in relation to production of the virgin material from which the recycled content is derived. This approach also does not assign environmental burdens to recycled materials that leave the product system. In other words, the avoided production of virgin material is here considered proportional to the "recycled content", i.e. the fraction of material in the input to the production that has been recycled in a previous system. In essence, this constitutes a form of allocation (Frischknecht 2010; ADEME 2007; BSI 2011). 
The "50/50 allocation" approach splits the burdens and benefits of recycling (i.e. the avoided inventory of virgin material production) equally between the product using recycled material input and the product at EoL providing the recycled material (Nicholson et al. 2009; AFNOR 2011).

For the EC OEF method, the choice of approach was based on the following (non-exhaustive) considerations, in particular to ensure alignment with criterion 1 (coverage of all life cycle stages) and criterion 4 (maximise physical representativeness):

- Whether (part of) the product has been recycled in a previous system. In such cases, a rate $\left(R_{1}\right)$ is used to define the "recycled content", i.e. the proportion of material in the input to the production that has been recycled in a previous system;

- Whether (part of) the product is assumed to be recycled in a subsequent product system. In such cases, a rate $\left(\mathrm{R}_{2}\right)$ is used to define the "recyclability" and credits are given against the (assumed) avoided production of virgin material;

- Whether the system includes an energy recovery step (e.g. thermal treatment with energy recovery). In such cases, a rate $\left(\mathrm{R}_{3}\right)$ is use to define the "energy recoverability rate" and credits are given against the recovered energy. Other parameters need also be included in the calculation of overall emissions, for example, the efficiency of the energy recovery process. Also, in case of energy recovery, the way of accounting for the substituted energy source (e.g. national average or specific mix) is crucial;

- Whether the recycled materials maintain the same intrinsic properties as the virgin material.

The approach recommended for the EC OEF method departs from the reviewed methods by incorporating all of the above mentioned aspects. To meet the third criterion of "reproducibility and comparability" a single approach, represented by a single, specific formula for all possible EoL scenarios (covering both closed-loop and open-loop recycling), was also deemed to be desirable. Towards this end, the "50/50 allocation" approach used in the BP X 30-323-0 method for open-loop recycling where "the market shows no visible disequilibrium (lack of secondary raw materials) [...]" (AFNOR 2011, p. 19) was adapted. Specifically, the adapted formula required for use in the EC OEF method is able to differentiate between recycled content $\left(R_{1}\right)$ and recyclability $\left(R_{2}\right)$ at EoL, to consider down-cycling, and to consider different disposal impacts of the products in a cascade product system (for a detailed description see European Commission 2013b and Allacker et al. (2013)). It should be noted that this approach satisfies criterion 4 at the system level, but not at the product level.

\subsection{Data quality requirements}

Although most of the methods evaluated do not specify minimum data quality requirements, some provide recommendations and guidance (Table 12). For example, ISO14064-3 stipulates a process for validation and verification of reported GHG emissions data, depending on the level of assurance required by the user of the data/intended application. Assessing the completeness, consistency, accuracy, and transparency of the provided information is required, with the objective to determine whether errors, omissions, and misinterpretations could affect the user's decisions.

The issue of data quality is particularly important with respect to criteria 3 (reproducibility/comparability) and 4 (physical representativeness). As a result, the EC OEF method necessarily goes further than the reviewed methodologies in order to ensure that data quality objectives are met.

We identified three related data quality issues that must be considered, and for which we deemed methodological specification to be necessary:

- Requirement for a data quality assessment (leading to data quality indicators)

- Requirements for data quality (i.e. minimum thresholds)

- Requirements for documentation and reporting of data quality assessments and indicators

To meet the criterion of reproducibility and comparability (criterion 3), it is insufficient to simply derive and report data quality indicators via a data quality assessment. This is because, despite that transparency with respect to data quality is increased, deriving conclusions on the basis of poor and/or uncertain data remains possible. Instead, these steps must be complemented with minimum data quality requirements. Towards this end, the EC OEF method establishes a mandatory, semi-quantitative data quality assessment scheme, with defined quality criteria with respect to: technological representativeness; geographical representativeness; temporal representativeness; completeness; parameter uncertainty; and methodological appropriateness and consistency. Additional documentation, nomenclature, and review requirements are also specified (European Commission 2010b).

While the goal is total reproducibility/comparability between EC OEF studies, which requires parity in data quality, in reality achieving perfect data representativeness will rarely be achievable. In order to balance feasibility and robustness, the EC OEF method takes a stepwise approach to data quality requirements. Specifically, "good quality" data (as determined via a semi-quantitative data quality assessment following the defined criteria) are required for data representing at least $70 \%$ of the environmental impacts caused by the organisation 
(in each environmental impact category considered). Lower quality requirements (as assessed based on expert judgement) apply for the data representing activities responsible for the remaining $30 \%$ of environmental impacts (of which $10 \%$ may be satisfied using "proxy data" in cases of data gaps). A detailed assessment scheme is provided.

\section{Conclusions and recommendations}

To date, a variety of methods and standards (or drafts thereof) have emerged for quantifying the environmental performance of organisations. However, on the basis of our analysis we suggest that methodological guidance for organisation environmental footprint studies is considerably less advanced and prescriptive than for product-level environmental footprint studies. In general, there is an apparent emphasis on how to report rather than how to calculate organisation environmental performance. Moreover, flexibility with respect to methodological norms is more common than are prescriptive requirements. Some methods do not require considering the entire life cycle of organisational activities, and most focus on only single indicators - both of which increase the possibility for burdenshifting. Nowhere did we identify recommended norms that were common across all reviewed methods, but in some cases, several of the methods were in agreement and were found to be suitable for adoption or adaptation. We therefore often found it necessary to provide methodological requirements that go beyond those of the reviewed methods. This is because the EC OEF method must be suitable to support a variety of potential applications, for which adherence to the four identified core criteria may be essential.

We recognise that meeting these more prescriptive methodological requirements - in particular, with respect to assessing a comprehensive range of potential environmental impacts and to data quality - has both costs and benefits. On the one hand, greater demands for technical knowledge (in support of data quality requirements) may increase the need for expertise and associated costs for organisations. On the other hand, a benefit of this more detailed and prescriptive approach is that less methodological expert knowledge will be required to apply the suite of more stringent methodological requirements because the user does not need to have the mature expertise in methodological matters that is necessary to support informed choices. Given that the target audience is not restricted to Life Cycle Assessment (LCA) experts, but rather encompasses environmental and technical managers in general who will not necessarily have an extensive background in LCA, this is a non-trivial consideration for the EC OEF method.

Our hope is that, overall, widespread adoption of the EC OEF method will lead to net cost savings for organisations. This optimism stems from the fact that the current pressures on organisations to comply with different methods, labelling and verification requirements come at significant expense. This reality is well recognized within the business community. In a public consultation on possible policy initiatives linked to the EC Product Environmental Footprint (PEF) and OEF methods, $72.5 \%$ of respondents identified the current lack of consistency as the most important barrier to the display and benchmarking of environmental performance (EC 2013c). Specific reasons included the existence of multiple initiatives in the EU $(70.8 \%)$ and the multiple ways of reporting $(76.3 \%)$.

The applicability of the EC OEF method has been tested in ten pilot cases covering a wide variety of sectors. The recommendations derived from these pilots were taken into account in the development of the final version of the EC OEF method. During the next 3 years, a second round of pilots will take place, focusing on the development of Organisation Environmental Footprint Sector Rules (OEFSRs). OEFSRs will provide sector-specific requirements that cannot be accommodated in the context of the general EC OEF method (for example, specifications regarding required system boundaries for downstream modelling, allocation solutions and supporting data, or required environmental impact categories). Specific emphasis during this 3 -year pilot phase will be placed on making the OEF process easier and less costly for SMEs by providing supporting tools, further simplified procedures, and by testing innovative approaches for data gathering. Other supply chain stakeholders will also be encouraged to be attentive to the particular needs of SMEs in this context.

Although the specific details of the actual policy contexts in which the EC OEF method will be applied are not yet fully resolved, the European Commission has recently adopted a Recommendation on the "Use of common methods to measure, benchmark and communicate the environmental performance of products and organisations" (EC 2013c). This Recommendation promotes the use of both the EC PEF and OEF methods, which form an integral part of the Recommendation. The Recommendation also includes a non-exhaustive, illustrative list of potential fields of application and takes duly into account the special needs of SMEs. Within the next 3 years, incorporating the methods in the Eco-Management and Audit Scheme (EMAS), Green Public Procurement, and in the EU Ecolabel is foreseen. Specific recommendations are also included regarding the use of the EC PEF and OEF methods in Member State policies, by companies and other private organisations, and by the financial community.

In conclusion, we suggest that formulating the EC OEF method with attention to the four overarching criteria has enabled us to arrive at a robust method for measuring, tracking, and reporting the environmental performance of organisations. The method represents a significant step forward for life-cycle based, multi-criteria environmental accountancy that provides 
for both enhanced reproducibility/consistency and physical representativeness. The end result, we believe, will contribute to better supporting our important, common goal of increasing the sustainability of production and consumption.

Acknowledgments Financial support for this work was provided under Administrative Arrangement (no. N70307/2009/552517, including Amendment No 1 from December 2010.) with the European Commission Directorate General-Environment. The contributions of the companies and other stakeholders participating in both the pilot studies and consultations for development of the EC OEF method are gratefully acknowledged.

Open Access This article is distributed under the terms of the Creative Commons Attribution License which permits any use, distribution, and reproduction in any medium, provided the original author(s) and the source are credited.

\section{References}

ADEME (2007) Bilan Carbone Companies and Local Authorities Version. Methodological Guide Version 5.0: objectives and principles for the counting of greenhouse gas emissions. French Agency for the Environment and Energy Management, Paris

AFNOR (2011) Repository of good practices. General principles for an environmental communication on mass market products. Part 0: General principles and methodological framework. AFNOR, Paris

Allacker K, Mathieux F, Manfredi S, Pelletier N, De Camillis C, Ardente F, Pant R (2013) Allocation solutions for secondary material production and end of life recovery: proposals for product policy initiatives. Resour Conserv Recy (In review)

Ascui F, Lovell H (2011) Carbon accounting and the construction of competence. J Clean Prod 36:48-59

Brander M, Wylie C (2011) The use of substitution in attributional life cycle assessment. GHG Measure Manage 1(3-4):161-166

BSI (2011) Specifications for the assessment of the life cycle GHG emissions of goods and services. http://www.bsigroup.com/en/ Standards-and-Publications/How-we-can-help-you/ProfessionalStandards-Service/PAS-2050/. Accessed July 2010

CDP (2010a) Carbon Disclosure Project. Information Request Guide. Carbon Disclosure Project, UK

CDP (2010b) Carbon Disclosure Project-Information Request Guide. CDP Water Disclosure, UK

DEFRA (2009) Guidance on how to measure and report your greenhouse gas emissions. Department for Environment, Food and Rural Affairs, London

Ekvall T, Finnveden G (2001) Allocation in ISO 14041-a critical review. J Clean Prod 9(3):197-208

European Commision-Directorate General-Environment (2012) Consultation on delivering more sustainable production and consumption. European Commission, Brussels, http://ec.europa.eu/ environment/consultations/sustainable.htm. Accessed April 2013

European Commission (2011a) Communication from the Commission to the European Parliament, the Council, the European Economic and Social Committee and the Committee of the Regions. A resource-efficient Europe-flagship initiative under the Europe 2020 Strategy. European Commission, Brussels

European Commission (2011b) Communication from the Commission to the European Parliament, the Council, the European Economic and Social Committee and the Committee of the Regions. Roadmap to a resource efficient Europe. European Commission, Brussels
European Commission (2013a) Annex II-Product Environmental Footprint (PEF) Guide to the COMMISSION RECOMMENDATION on the use of common methods to measure and communicate the life cycle environmental performance of products and organisations. http:// ec.europa.eu/environment/eussd/smgp/pdf/annex2_recommendation. pdf. Accessed April 2013

European Commission (2013b) Annex III - Organisational Environmental Footprint (OEF) Guideto the COMMISSION RECOMMENDATION on the use of common methods to measure and communicate the life cycle environmental performance of products and organisations . http:// ec.europa.eu/environment/eussd/smgp/pdf/annex3_recommendation. pdf. Accessed April 2013

European Commission (2013c) Communication from the Commission to the European Parliament and the Council. Building a single market for green products: facilitating better methods to measure, benchmark and communicate the environmental performance of products and organisations. http://ec.europa.eu/environment/ eussd/smgp/pdf/communication.pdf. Accessed April 2013

European Commission-Joint Research Centre-Institute for Environment and Sustainability (2010a) International Reference Life Cycle Data System (ILCD) Handbook - general guide for life cycle assessment — detailed guidance. Publications Office of the European Union, Luxembourg, First edition March 2010. EUR 24708 EN

European Commission-Joint Research Centre-Institute for Environment and Sustainability (2010b) International Reference Life Cycle Data System (ILCD) Handbook - review schemes for life cycle assessment. Publications Office of the European Union, Luxembourg, First edition March 2010. EUR 24710 EN

European Commission-Joint Research Centre-Institute for Environment and Sustainability (2010c) International Reference Life Cycle Data System (ILCD) Handbook - framework and requirements for life cycle impact assessment models and indicators. Publications Office of the European Union, Luxembourg, First edition March 2010. EUR 24586 EN

European Commission-Joint Research Centre-Institute for Environment and Sustainability (2010d) International Reference Life Cycle Data System (ILCD) Handbook - specific guide for life cycle inventory data sets. Publications Office of the European Union, Luxembourg, First edition. EUR 24709 EN

European Commission-Joint Research Centre-Institute for Environment and Sustainability (2011a) International Reference Life Cycle Data System (ILCD) Handbook - recommendations based on existing environmental impact assessment models and factors for Life Cycle Assessment in a European context. Publications Office of the European Union

European Commission-Joint Research Centre-Institute for Environment and Sustainability (2011b) Organisational Environmental Footprint Guide - Draft. http://ec.europa.eu/environment/eussd/corporate footprint.htm. Accessed August 2012

European Commission-Joint Research Centre-Institute for Environment and Sustainability (2011c) Analysis of existing environmental footprint methodologies for products and organisations: recommendations, rationale, and alignment. http://ec.europa.eu/environment/ eussd/pdf/Deliverable.pdf. Accessed August 2012

Frischknecht R (2010) LCI modelling approaches applied on recycling of materials in view of environmental sustainability, risk perception and eco-efficiency. Int J Life Cycle Assess 15:666-671

GRI (2006) Sustainability reporting guidelines (G3). Global Reporting Initiative, Amsterdam

Guinée J et al (2002) Handbook on life cycle assessment. Operational guide to the ISO standards. I: LCA in perspective. IIa: Guide. IIb: Operational annex. III: Scientific background. Kluwer Academic Publishers, Netherlands

ISO (2006a) ISO 14044. Environmental management-life cycle assessment-requirements and guidelines. International Organization for Standardization, Geneva 
ISO (2006b) ISO 14064-1. Greenhouse gases-Part 1: Specification with guidance at the organization level for quantification and reporting of greenhouse gas emissions and removals. International Organization for Standardization, Geneva

ISO (2006c) ISO 14064-3. Greenhouse gases-Part 3: Specification with guidance at the validation and verification of greenhouse gas assertions. International Organization for Standardization, Geneva

ISO/WD TR 14069 working draft 2 (2010) Greenhouse gases (GHG) - Quantification and reporting of GHG emissions for organizations (Carbon footprint of organization) - Guidance for the application of ISO 14064-1, under development

Moneva J, Archel P, Correa C (2006) GRI and the camouflaging of corporate unsustainability. Account Forum 30(2):121-137

Nicholson A, Olivetti E, Gregory J, Field F, Kirchain R (2009) End-oflife allocation methods: open loop recycling impacts on robustness of material selection decisions. Proc Int Symp Sust Syst Technol 18-20:1-6. doi:10.1109/ISSST.2009.5156769

Pelletier N, Tyedmers P (2011) An ecological economic critique of the use of market information in life cycle assessment research. J Ind Ecol 15(3):342-354
Pelletier N, Tyedmers P (2012) Response to Weinzettel. J Ind Ecol 16(3):456-458

Sangwon S, Lenzen M, Treloar G, Hondo H, Horvath A, Huppes G, Jolliet O, Klann U, Krewitt W, Moriguchi Y, Munksgaard J, Norris G (2004) System boundary selection in life-cycle inventories using hybrid approaches. Environ Sci Technol 38(3):657664

Schaltegger S, Burrit R (2010) Sustainability accounting for companies; Catchphrase or decision support for business leaders? J World Bus 45:375-384

Wardenaar T, van Ruijven T, Mendoza Beltran A, Vad K, Guinee J, Heijungs R (2012) Differences between LCA for analysis and LCA for policy: a case study on the consequences of allocation choices in bio-energy policies. Int J LCA 17(8):1059-1067

WRI and WBCSD (2004) GHG Protocol Corporate Accounting and Reporting Standard. World Resources Institute, Washington, DC

WRI and WBCSD (2011) Corporate Value Chain (Scope 3) Accounting and Reporting Standard-Supplement to the GHG Protocol Corporate Accounting and Reporting Standard. World Resources Institute, Washington, DC 